\title{
Atomic data for astrophysics: Fe XII soft X-ray lines ${ }^{\star}$
}

\author{
G. Del Zanna ${ }^{1}$, P. J. Storey ${ }^{2}$, N. R. Badnell ${ }^{3}$, and H. E. Mason ${ }^{1}$ \\ 1 DAMTP, Centre for Mathematical Sciences, Wilberforce Road, Cambridge, CB3 0WA, UK \\ e-mail: g.del-zanna@damtp.cam.ac.uk \\ 2 Department of Physics and Astronomy, University College London, Gower Street, London, WC1E 6BT, UK \\ 3 Department of Physics, University of Strathclyde, Glasgow, G4 0NG, UK
}

Received 12 February 2012 / Accepted 30 May 2012

\begin{abstract}
We present new large-scale R-matrix (up to $n=4$ ) and distorted-wave (DW, up to $n=6$ ) scattering calculations for electron collisional excitation of Fe xiI. The first aim is to provide accurate atomic data for the soft X-rays, where strong decays from the $n=4$ levels are present. As found in previous work on Fex, resonances attached to $n=4$ levels increase the cross-sections for excitations from the ground state to some $n=4$ levels, when compared to DW calculations. Cascading from higher levels is also important. We provide a number of models and line intensities, and list a number of strong unidentified lines. The second aim is to assess the effects of the large R-matrix calculation on the $n=3$ transitions. Compared to our previous $(n=3) \mathrm{R}$-matrix calculation, we find overall excellent agreement to within a few percent, however a few key density diagnostic EUV intensities differ by about $60 \%$ at coronal densities. The new atomic data result in lower electron densities, resolving previous discrepancies with solar observations.
\end{abstract}

Key words. atomic data - line: identification - techniques: spectroscopic - Sun: corona

\section{Introduction}

The soft X-ray (50-170 ̊) spectrum of the quiet and active Sun is rich in $n=4 \rightarrow n=3$ transitions from highly ionised iron ions, from Fe viI to Fe xvi (see, e.g. Fawcett et al. 1968; Manson 1972; Behring et al. 1972). Very little atomic data are currently available for these ions and the majority of the spectral lines still await firm identification, despite the fact that various current missions are routinely observing the soft X-rays. The Solar Dynamic Observatory (SDO) Atmospheric Imaging Assembly (AIA, see Lemen et al. 2012) and the Extreme ultraviolet Variability Experiment (EVE) (Woods et al. 2012) have been observing the Sun in the soft X-rays. Soft X-ray spectra of stellar coronae are also routinely observed with the Chandra Low Energy Transmission Grating spectrometer (LETG, see Brinkman et al. 2000).

The atomic data for Fe vIII and Fe IX relevant for the soft X-rays have recently been discussed in O'Dwyer et al. (2012), where new distorted wave (DW) scattering calculations for these two ions were presented. The main issues related to calculating accurate atomic data for the $n=4$ levels are discussed in Del Zanna et al. (2012), where new atomic data for Fe $\mathrm{x}$ have been presented.

Here, we present new large-scale scattering calculations for the Fe XII soft X-ray lines. We also review the extreme-ultraviolet (EUV) lines, given their importance for many current missions, especially for the Hinode EUV Imaging Spectrometer (EIS, see Culhane et al. 2007) where Fe XII lines are prominent in its two

* The full dataset (energies, transition probabilities and rates) are available in electronic form at our APAP website

(http://www.apap-network.org) as well as at the CDS via anonymous ftp to cdsarc.u-strasbg. fr $(130.79 .128 .5)$ or via http://cdsarc.u-strasbg.fr/viz-bin/qcat? J/A+A/543/A139 spectral ranges (SW: 166-212 ̊; LW: 245-291 ̊). Fe xII lines are routinely used to measure electron densities, in particular the ratio of the two self-blends at 186.88 and $195.12 \AA$. These strong lines provide the best density diagnostic for the quiet solar corona for this ion. It has been pointed out by many authors (see, e.g. Young et al. 2009) that these diagnostics produce higher densities than those obtained with other ions formed close in temperature. A full benchmark of the coronal density diagnostics available to Hinode EIS was performed by Del Zanna (2012), where these inconsistencies, although not very large, were confirmed. We therefore aim to investigate whether a larger calculation can resolve these discrepancies.

This paper is organised as follows. In Sect. 2, we give a brief review of previous observations and atomic calculations. In Sect. 3 we outline the methods adopted for the scattering calculations. In Sect. 4 we present our results and in Sect. 5 we reach our conclusions.

\section{Previous observations and atomic data for Fe XII}

A history of the identifications of all the EUV and visible forbidden lines (from within the $n=3$ configurations) for Fe XII was given in detail in Del Zanna \& Mason (2005) and is not repeated here. Historically, large discrepancies between observed and predicted intensities of EUV transitions from the $n=3$ levels were found to be present. These discrepancies were finally resolved by Storey et al. (2005) via an R-matrix electron collisional calculation carried-out within the Iron Project. References to earlier calculations for the $n=3$ levels are given in Storey et al. (2005).

The configuration basis describing the target adopted by Storey et al. (2005) included 12 of the main $n=3$ configurations. For the scattering calculation the lowest $58 \mathrm{LS}$ terms were included, giving rise to 143 fine-structure levels. The expansion of 
each scattered electron partial wave was over a basis of 20 functions within the R-matrix boundary, and the partial wave expansion extended to a maximum total orbital angular momentum quantum number of $L=18$. The outer region calculation was carried out using the intermediate-coupling frame transformation method (ICFT) described by Griffin et al. (1998), up to a total angular momentum quantum number $J=15$. The nonexchange calculation extended from $J=16$ to $J=50$.

Del Zanna \& Mason (2005) complemented the Storey et al. (2005) collisional rates with a set of $A$-values. We refer to this ion model here as DM05. Del Zanna \& Mason (2005) benchmarked line intensities against various experimental data. Excellent agreement was found, and a large number of new identifications were proposed. These new observed energies are adopted here. These data have been used since 2005 within the CHIANTI database (Dere et al. 1997; Landi et al. 2006). Later, Del Zanna (2012) used Hinode EIS observations to confirm most of the new identifications.

For the soft X-rays ( $n=4 \rightarrow n=3$ transitions), the identifications of some of the $3 \mathrm{~s}^{2} 3 \mathrm{p}^{2} 4 l(l=\mathrm{s}, \mathrm{p}, \mathrm{d}, \mathrm{f})$ levels are due to the fundamental laboratory work by Fawcett et al. (1972). It is important to keep in mind that only lines with strong oscillator strengths were identified, that some identifications were tentative, and that a large number of lines in the spectra were left unidentified. We have re-analysed some of Fawcett's plates as part of a larger project to sort out the identifications in the Fe soft X-ray spectrum.

\section{Methods}

The atomic structure calculations were carried out using the AUTOSTRUCTURE program (Badnell 1997) which constructs target wavefunctions using radial wavefunctions calculated in a scaled Thomas-Fermi-Dirac statistical model potential with a set of scaling parameters. The Breit-Pauli distorted wave calculations were carried out using the recent development of the AUtostructure code, described in detail in Badnell (2011). Collision strengths are calculated at the same set of final scattered energies for all transitions. "Top-up" for the contribution of high partial waves is done using the same Breit-Pauli methods and subroutines implemented in the R-matrix outer-region code STGF. The program also provides radiative rates and infinite energy Born limits. These limits are particularly important for two aspects. First, they allow a consistency check of the collision strengths in the scaled Burgess \& Tully (1992) domain (see also Burgess et al. 1997). Second, they are used in the interpolation of the collision strengths at high energies when calculating the Maxwellian averages.

The R-matrix method used in the inner region of the scattering calculation is described in Hummer et al. (1993) and Berrington et al. (1995). We performed it in $L S$ coupling and included the mass-velocity and Darwin relativistic operators. The outer region calculation used the ICFT method (Griffin et al. 1998). Dipole-allowed transitions were topped-up to infinite partial wave using an intermediate coupling version of the Coulomb-Bethe method as described by Burgess (1974) while non-dipole allowed transitions were topped-up assuming that the collision strengths form a geometric progression in $J$ (see Badnell \& Griffin 2001). The collision strengths were extended to high energies by interpolation using the appropriate highenergy limits in the Burgess \& Tully (1992) scaled domain. The high-energy limits were calculated with AUTOSTRUCTURE for both optically-allowed (see Burgess et al. 1997) and non-dipole allowed transitions (see Chidichimo et al. 2003). The temperature- dependent effective collisions strength $\Upsilon(i-j)$ were calculated by assuming a Maxwellian electron distribution and linear integration with the final energy of the colliding electron.

\section{Results}

Several calculations have been performed with different size target expansions and corresponding ion population models have been constructed to predict line intensities and compare with observations. A summary of our investigations is presented here.

We started with various DW calculations systematically increasing the number of configurations up to and including those with $n=6$ valence orbitals. The DW rates for the higher levels were supplemented with the Storey et al. (2005) R-matrix data, to ensure that the metastable levels are correctly populated.

We then performed separate structure calculations for each ion model to calculate all of the radiative data for all transitions among the levels. This ensures that all the cascading from the target configurations is included. We then calculated the level populations and the relative line intensities so as to find out which lines are expected to be strongest. We then compared the results to the laboratory plates of Fawcett and to solar spectra.

As in the Fe x case (Del Zanna et al. 2012), we found large discrepancies between observed and predicted intensities, in particular for the decays from the $3 s^{2} 3 p^{2} 4$ s levels. Exactly as in the Fe $x$ case, we also predicted some (unidentified) decays from the $3 \mathrm{~s} 3 \mathrm{p}^{3} 4 \mathrm{~s}$ levels to be even stronger, in coronal conditions, than the decays from the $3 \mathrm{~s}^{2} 3 \mathrm{p}^{2} 4 \mathrm{~s}$ levels (as we will show below).

We followed the procedure outlined in the Fe $\mathrm{x}$ case to estimate which configurations would be likely to be producing resonances in the collision strengths for the spectroscopically important configurations/levels. For Fe $\mathrm{x}$, we found that the excitations to the $3 s^{2} 3 p^{4} 4$ s levels are significantly underestimated by the DW calculations, mostly because of resonances due to the $3 s^{2} 3 p^{4} 4 p$ levels. Similarly, we also found the $3 s^{2} 3 p^{2} 4 s$ levels in Fe XII to be affected by the same issues. We found that most resonance excitations to the $n=4$ levels come from a number of configurations which we have then included in the scattering calculation.

\subsection{The R-matrix and DW calculations for the $n=3,4$ levels}

As our configuration basis set we have chosen the complete set of 36 configurations shown in Fig. 1 and listed in Table 1. The scaling parameters $\lambda_{n l}$ for the potentials in which the orbital functions are calculated are also given in Table 1. A full R-matrix calculation with all the $n=4$ levels is prohibitive at present, because it would involve 1538 LS terms and 4071 levels. For the scattering close-coupling calculation, we retained 912 finestructure levels arising from the first $356 L S$ terms of the lowest set of 20 configurations (see Fig. 1). We have performed both an ICFT R-matrix and a DW calculation using the same basis. They have both been challenging large-scale calculations.

Table 2 presents a selection of fine-structure target level energies $E_{\mathrm{t}}$, compared to experimental energies $E_{\text {exp }}$. The latter have been obtained from Del Zanna \& Mason (2005) for the $n=3$ levels, otherwise from a selection of Fawcett et al. (1972) identifications which we assessed individually.

There is good overall agreement in terms of energy differences between levels. A set of "best" energies $E_{\mathrm{b}}$ was obtained with a quadratic fit between the $E_{\exp }$ and $E_{\mathrm{t}}$ values. The $E_{\mathrm{b}}$ values were used (together with the $E_{\exp }$ ones) within the R-matrix calculation to obtain an accurate position of the resonance 


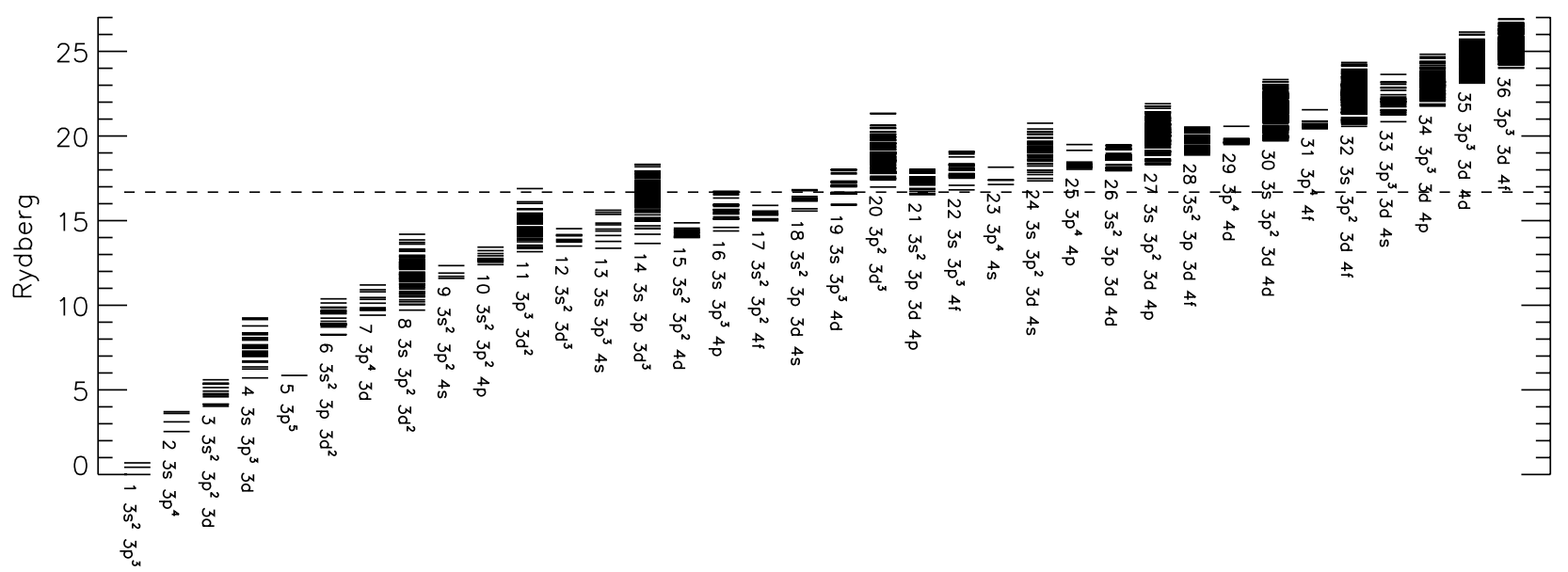

Fig. 1. Term energies of the target levels (36 configurations) for the $n=4$ calculations. The 356 terms which produce levels having energies below the dashed line have been retained for the close-coupling expansion.

Table 1. Target electron configuration basis and orbital scaling parameters $\lambda_{n l}$ for the R-matrix and DW runs.

\begin{tabular}{l|cc}
\hline \hline Configurations & & $\lambda_{n l}$ \\
\hline $3 \mathrm{~s}^{2} 3 \mathrm{p}^{3}$ & $1 \mathrm{~s}$ & 1.41176 \\
$3 \mathrm{~s}^{2} 3 \mathrm{p}^{2} 3 \mathrm{~d}$ & $2 \mathrm{~s}$ & 1.11813 \\
$3 \mathrm{~s}^{2} 3 \mathrm{p} 3 \mathrm{~d}^{2}$ & $2 \mathrm{p}$ & 1.06218 \\
$3 \mathrm{~s}^{2} 3 \mathrm{p}^{2} 4 l(l=\mathrm{s}, \mathrm{p}, \mathrm{d}, \mathrm{f})$ & $3 \mathrm{~s}$ & 1.13366 \\
$3 \mathrm{~s}^{2} 3 \mathrm{p} 3 \mathrm{~d} 4 l(l=\mathrm{s}, \mathrm{p}, \mathrm{d}, \mathrm{f})$ & $3 \mathrm{p}$ & 1.10544 \\
$3 \mathrm{~s}^{2} 3 \mathrm{~d}^{3}$ & $3 \mathrm{~d}$ & 1.11782 \\
$3 \mathrm{~s} 3 \mathrm{p}^{4}$ & $4 \mathrm{~s}$ & 1.16349 \\
$3 \mathrm{~s} 3 \mathrm{p}^{3} 3 \mathrm{~d}$ & $4 \mathrm{p}$ & 1.13150 \\
$3 \mathrm{~s} 3 \mathrm{p}^{3} 4 l(l=\mathrm{s}, \mathrm{p}, \mathrm{d}, \mathrm{f})$ & $4 \mathrm{~d}$ & 1.15366 \\
$3 \mathrm{~s} 3 \mathrm{p}^{2} 3 \mathrm{~d}^{2}$ & $4 \mathrm{f}$ & 1.31107 \\
$3 \mathrm{~s} 3 \mathrm{p}^{2} 3 \mathrm{~d} 4 l(l=\mathrm{s}, \mathrm{p}, \mathrm{d}, \mathrm{f})$ & $5 \mathrm{~s}$ & 1.17570 \\
$3 \mathrm{~s} 3 \mathrm{p} 3 \mathrm{~d}^{3}$ & $5 \mathrm{p}$ & 1.13910 \\
$3 \mathrm{~s} 3 \mathrm{p} 3 \mathrm{~d}^{3}$ & $5 \mathrm{~d}$ & 1.16936 \\
$3 \mathrm{p}^{5}$ & $5 \mathrm{f}$ & 1.33627 \\
$3 \mathrm{p}^{4} 3 \mathrm{~d}$ & $5 \mathrm{~g}$ & 1.56139 \\
$3 \mathrm{p}^{4} 4 l(l=\mathrm{s}, \mathrm{p}, \mathrm{d}, \mathrm{f})$ & $6 \mathrm{~s}$ & 1.17811 \\
$3 \mathrm{p}^{3} 3 \mathrm{~d}^{2}$ & $6 \mathrm{p}$ & 1.14207 \\
$3 \mathrm{p}^{3} 3 \mathrm{~d} 4 l(l=\mathrm{s}, \mathrm{p}, \mathrm{d}, \mathrm{f})$ & $6 \mathrm{~d}$ & 1.16624 \\
$3 \mathrm{p}^{2} 3 \mathrm{~d}^{3}$ & $6 \mathrm{f}$ & 1.29974 \\
\hline $3 \mathrm{~s}^{2} 3 \mathrm{p}^{2} 5 l(l=\mathrm{s}, \mathrm{p}, \mathrm{d}, \mathrm{f}, \mathrm{g})$ & $6 \mathrm{~g}$ & 1.45339 \\
$3 \mathrm{~s} 3 \mathrm{p}^{3} 5 l(l=\mathrm{s}, \mathrm{p}, \mathrm{d}, \mathrm{f}, \mathrm{g})$ & & \\
$3 \mathrm{p}^{4} 5 l(l=\mathrm{s}, \mathrm{p}, \mathrm{d}, \mathrm{f}, \mathrm{g})$ & & \\
$3 \mathrm{~s}^{2} 3 \mathrm{p}^{2} 6 l(l=\mathrm{s}, \mathrm{p}, \mathrm{d}, \mathrm{f}, \mathrm{g})$ & & \\
$3 \mathrm{~s} 3 \mathrm{p}^{3} 6 l(l=\mathrm{s}, \mathrm{p}, \mathrm{d}, \mathrm{f}, \mathrm{g})$ & & \\
$3 \mathrm{p}^{4} 6 l(l=\mathrm{s}, \mathrm{p}, \mathrm{d}, \mathrm{f}, \mathrm{g})$ & & \\
\hline
\end{tabular}

Notes. The configurations below the line were used for the DW calculation DW6.

thresholds. They were also used to calculate the transition probabilities, which were computed separately using the full $n=4$ target basis. We then built two ion population models, using these transition probabilities, one with the R-matrix rates (hereafter RM4) and one with just the DW rates (hereafter DW4).

The expansion of each scattered electron partial wave was carried out over a basis of 19 functions within the R-matrix boundary and the partial wave expansion extended to a maximum total orbital angular momentum quantum number of $L=16$. This produces accurate collision strengths up to about 80 Ryd.
The outer region calculation includes exchange up to a total angular momentum quantum number $J=26 / 2$. We have supplemented the exchange contributions with a non-exchange calculation extending from $J=28 / 2$ to $J=74 / 2$. The outer region exchange calculation was performed in a number of stages. A coarse energy mesh was chosen above all resonances. The resonance region itself was calculated with an increasing number of energies to check for convergence, as was done for the Iron Project Fe xi calculation (Del Zanna et al. 2010). The results presented here are obtained with 8800 points in the resonance region.

We then compared the collision strengths and their thermal averages with the DW results and those by Storey et al. (2005) for the $n=3$ levels. The comparisons for a selection of levels giving rise to important transitions are displayed in Figs. 2-7. Excellent agreement between the background R-matrix and the DW collision strengths is found in all cases. This is to be expected since they both use the same target. Excellent agreement is found with the Storey et al. (2005) data for all the dipoleallowed transitions (e.g. Fig. 7). However, significant increases are found for a number of forbidden transitions. We will return to this issue below.

We then looked at the transitions to the $n=4$ levels. Again, good agreement between the R-matrix background and the DW data is found. However, as we expected, we find significant resonance contribution for transitions to a number of levels, in particular to the $3 \mathrm{~s}^{2} 3 \mathrm{p}^{2} 4 \mathrm{~s}$ ones. Figure 8 shows the collision strengths for the $79.5 \AA$ transition as an example. The enhancements are not as strong as we found in the Fe x case, but become increasingly important at low temperatures.

\subsection{The $n=3$ main EUV density diagnostics}

The relative intensities of the brightest lines from the $n=3$ levels at coronal densities are shown in Table 3. We show both the values obtained from the present RM4 model and from the previous DM05, based on the Storey et al. (2005) collision rates. For most cases, we obtain the same intensities with these two models. However, for the important density diagnostic lines we find significantly increased line intensities. In particular, the selfblend of the 3-39 and 2-36 transitions at $186.88 \AA$ (identified in Del Zanna \& Mason 2005) and the 3-34 one at $196.64 \AA$ are increased by $60 \%$. These two lines are the best electron density 
Table 2. Level energies for Fe XII $(n=3,4)$.

\begin{tabular}{|c|c|c|c|c|c|}
\hline$i$ & Conf. & Lev. & $E_{\text {exp }}$ & $E_{\mathrm{t}}$ & $E_{\mathrm{t}}(\mathrm{S} 05)$ \\
\hline 1 & $3 s^{2} 3 p^{3}$ & ${ }^{4} S_{3 / 2}$ & 0.0 & 0.0 & 0.0 \\
\hline 2 & $3 s^{2} 3 p^{3}$ & ${ }^{2} \mathrm{D}_{3 / 2}$ & 0.379 & $0.405(-0.027)$ & $0.405(-0.026)$ \\
\hline 3 & $3 s^{2} 3 p^{3}$ & ${ }^{2} \mathrm{D}_{5 / 2}$ & 0.420 & $0.447(-0.027)$ & $0.445(-0.025)$ \\
\hline 4 & $3 s^{2} 3 p^{3}$ & ${ }^{2} \mathrm{P}_{1 / 2}$ & 0.675 & $0.706(-0.031)$ & $0.698(-0.023)$ \\
\hline 5 & $3 s^{2} 3 p^{3}$ & ${ }^{2} \mathrm{P}_{3 / 2}$ & 0.734 & $0.762(-0.028)$ & $0.752(-0.019)$ \\
\hline 6 & $3 s 3 p^{4}$ & ${ }^{4} \mathrm{P}_{5 / 2}$ & 2.500 & $2.496(0.004)$ & $2.493(0.008)$ \\
\hline 7 & $3 \mathrm{~s} 3 \mathrm{p}^{4}$ & ${ }^{4} \mathrm{P}_{3 / 2}$ & 2.588 & $2.582(0.006)$ & $2.577(0.011)$ \\
\hline 8 & $3 s 3 p^{4}$ & ${ }^{4} \mathrm{P}_{1 / 2}$ & 2.627 & $2.622(0.006)$ & $2.616(0.011)$ \\
\hline 9 & $3 s 3 p^{4}$ & ${ }^{2} \mathrm{D}_{3 / 2}$ & 3.096 & $3.119(-0.024)$ & $3.113(-0.017)$ \\
\hline 10 & $3 \mathrm{~s} 3 p^{4}$ & ${ }^{2} \mathrm{D}_{5 / 2}$ & 3.114 & $3.137(-0.023)$ & $3.129(-0.015)$ \\
\hline 11 & $3 s^{2} 3 p^{2} 3 d$ & ${ }^{2} \mathrm{P}_{3 / 2}$ & 3.551 & $3.596(-0.044)$ & $3.591(-0.040)$ \\
\hline 12 & $3 s^{2} 3 p^{2} 3 d$ & ${ }^{2} \mathrm{P}_{1 / 2}$ & 3.594 & $3.641(-0.047)$ & $3.633(-0.039)$ \\
\hline 13 & $3 \mathrm{~s} 3 \mathrm{p}^{4}$ & ${ }^{2} \mathrm{~S}_{1 / 2}$ & 3.740 & $3.787(-0.047)$ & $3.773(-0.033)$ \\
\hline 14 & $3 s^{2} 3 p^{2} 3 d$ & ${ }^{4} \mathrm{~F}_{3 / 2}$ & 3.891 & $3.947(-0.056)$ & $3.945(-0.054)$ \\
\hline 15 & $3 s^{2} 3 p^{2} 3 d$ & ${ }^{4} \mathrm{~F}_{5 / 2}$ & 3.925 & $3.982(-0.057)$ & $3.979(-0.054)$ \\
\hline 16 & $3 s^{2} 3 p^{2} 3 d$ & ${ }^{4} \mathrm{~F}_{7 / 2}$ & 3.974 & $4.033(-0.059)$ & $4.029(-0.055)$ \\
\hline 17 & $3 s^{2} 3 p^{2} 3 d$ & ${ }^{4} \mathrm{~F}_{9 / 2}$ & 4.038 & $4.097(-0.059)$ & $4.091(-0.053)$ \\
\hline 18 & $3 s^{2} 3 p^{2} 3 d$ & ${ }^{2} \mathrm{~F}_{5 / 2}$ & - & 4.099 & 4.095 \\
\hline 19 & $3 s^{2} 3 p^{2} 3 d$ & ${ }^{4} \mathrm{D}_{1 / 2}$ & - & 4.129 & 4.122 \\
\hline 20 & $3 s^{2} 3 p^{2} 3 d$ & ${ }^{4} \mathrm{D}_{7 / 2}$ & 4.074 & $4.135(-0.061)$ & $4.129(-0.055)$ \\
\hline 21 & $3 s^{2} 3 p^{2} 3 d$ & ${ }^{4} \mathrm{D}_{3 / 2}$ & 4.074 & $4.140(-0.066)$ & $4.132(-0.058)$ \\
\hline 22 & $3 s^{2} 3 p^{2} 3 d$ & ${ }^{4} \mathrm{D}_{5 / 2}$ & 4.126 & $4.182(-0.057)$ & $4.174(-0.049)$ \\
\hline 23 & $3 s^{2} 3 p^{2} 3 d$ & ${ }^{2} \mathrm{~F}_{7 / 2}$ & 4.205 & $4.271(-0.066)$ & $4.262(-0.057)$ \\
\hline 24 & $3 s^{2} 3 p^{2} 3 d$ & ${ }^{2} \mathrm{G}_{7 / 2}$ & 4.506 & $4.598(-0.092)$ & $4.592(-0.085)$ \\
\hline 25 & $3 \mathrm{~s}^{2} 3 \mathrm{p}^{2} 3 \mathrm{~d}$ & ${ }^{2} \mathrm{G}_{9 / 2}$ & 4.531 & $4.626(-0.09$ & $4.619(-0.087)$ \\
\hline 26 & $3 \mathrm{~s} 3 \mathrm{p}^{4}$ & ${ }^{2} \mathrm{P}_{3 / 2}$ & 4.573 & $4.665(-0.092)$ & $4.649(-0.077)$ \\
\hline 27 & $3 s^{2} 3 p^{2} 3 d$ & ${ }^{4} \mathrm{P}_{5 / 2}$ & 4.670 & $4.764(-0.094)$ & $4.754(-0.084)$ \\
\hline 28 & $3 \mathrm{~s} 3 \mathrm{p}^{4}$ & ${ }^{2} \mathrm{P}_{1 / 2}$ & 4.683 & $4.772(-0.090)$ & $4.755(-0.073)$ \\
\hline 29 & $3 \mathrm{~s}^{2} 3 \mathrm{p}^{2} 3 \mathrm{~d}$ & ${ }^{4} \mathrm{P}_{3 / 2}$ & 4.709 & $4.801(-0.09$ & $4.790(-0.081)$ \\
\hline 30 & $3 s^{2} 3 p^{2} 3 d$ & ${ }^{4} \mathrm{P}_{1 / 2}$ & 4.736 & $4.825(-0.0$ & $4.813(-0.076)$ \\
\hline 31 & $3 \mathrm{~s}^{2} 3 \mathrm{p}^{2} 3 \mathrm{~d}$ & ${ }^{2} \mathrm{D}_{3 / 2}$ & 4.794 & $4.892(-0.0$ & $4.880(-0.086)$ \\
\hline 32 & $3 s^{2} 3 p^{2} 3 d$ & ${ }^{2} \mathrm{D}_{5 / 2}$ & 4.893 & $4.995(-0.1$ & $4.980(-0.087)$ \\
\hline 33 & $3 s^{2} 3 p^{2} 3 d$ & ${ }^{2} \mathrm{D}_{3 / 2}$ & 5.048 & $5.154(-0.1$ & $5.138(-0.090)$ \\
\hline 34 & $3 s^{2} 3 p^{2} 3 d$ & ${ }^{2} \mathrm{D}_{5 / 2}$ & 5.054 & 5.16 & $5.146(-0.092)$ \\
\hline 35 & $3 \mathrm{~s}^{2} 3 \mathrm{p}^{2} 3 \mathrm{~d}$ & ${ }^{2} \mathrm{P}_{1 / 2}$ & 5.192 & 5.31 & $5.309(-0.116)$ \\
\hline 36 & $3 \mathrm{~s}^{2} 3 \mathrm{p}^{2} 3 \mathrm{~d}$ & ${ }^{2} \mathrm{~F}_{5 / 2}$ & 5.256 & $5.384(-0.1$ & $5.370(-0.114)$ \\
\hline 37 & $3 s^{2} 3 p^{2} 3 d$ & ${ }^{2} \mathrm{~S}_{1 / 2}$ & 5.250 & $5.387(-0.1$ & $5.366(-0.115)$ \\
\hline 38 & $3 s^{2} 3 p^{2} 3 d$ & ${ }^{2} \mathrm{P}_{3 / 2}$ & 5.264 & 5.39 & $5.381(-0.1$ \\
\hline 39 & $3 s^{2} 3 p^{2} 3 d$ & ${ }^{2} \mathrm{~F}_{7 / 2}$ & 5.296 & 5.42 & $5.410(-0$ \\
\hline 40 & $3 \mathrm{~s}^{2} 3 \mathrm{p}^{2} 3 \mathrm{~d}$ & ${ }^{2} \mathrm{D}_{5 / 2}$ & 5.504 & 3) & $7(-0.114)$ \\
\hline 41 & $3 s^{2} 3 p^{2} 3 d$ & ${ }^{2} \mathrm{D}_{3 / 2}$ & 5.518 & 5.65 & $5.631(-0.113)$ \\
\hline 84 & $3 \mathrm{~s} 3 \mathrm{p}^{3} 3 \mathrm{~d}$ & ${ }^{4} \mathrm{D}_{7 / 2}$ & 7.295 & 76) & $7.456(-0.161)$ \\
\hline 85 & $3 s 3 p^{3} 3 d$ & ${ }^{2} \mathrm{D}_{3 / 2}$ & 7.339 & $7.479(-0.140)$ & $7.468(-0.130)$ \\
\hline 87 & $3 s 3 p^{3} 3 d$ & ${ }^{4} \mathrm{D}_{5 / 2}$ & 7.330 & $7.505(-0.175)$ & $7.489(-0.159)$ \\
\hline 272 & $3 s^{2} 3 p^{2} 4 s$ & ${ }^{4} \mathrm{P}_{1 / 2}$ & 11.318 & $11.505(-0.187)$ & - \\
\hline 278 & $3 s^{2} 3 p^{2} 4 s$ & ${ }^{4} \mathrm{P}_{3 / 2}$ & 11.388 & $11.567(-0.179)$ & - \\
\hline 288 & $3 s^{2} 3 p^{2} 4 s$ & ${ }^{4} \mathrm{P}_{5 / 2}$ & 11.464 & $11.639(-0.175)$ & - \\
\hline 290 & $3 s^{2} 3 p^{2} 4 s$ & ${ }^{2} \mathrm{P}_{1 / 2}$ & 11.461 & $11.655(-0.193)$ & - \\
\hline 298 & $3 s^{2} 3 p^{2} 4 s$ & ${ }^{2} \mathrm{P}_{3 / 2}$ & 11.540 & $11.739(-0.199)$ & - \\
\hline 324 & $3 s^{2} 3 p^{2} 4 s$ & ${ }^{2} \mathrm{D}_{5 / 2}$ & 11.734 & $11.935(-0.200)$ & - \\
\hline 325 & $3 s^{2} 3 p^{2} 4 s$ & ${ }^{2} \mathrm{D}_{3 / 2}$ & 11.747 & $11.948(-0.201)$ & - \\
\hline 364 & $3 s^{2} 3 p^{2} 4 p$ & ${ }^{4} \mathrm{D}_{3 / 2}$ & 12.296 & $12.472(-0.176)$ & - \\
\hline 370 & $3 s^{2} 3 p^{2} 4 p$ & ${ }^{4} \mathrm{D}_{5 / 2}$ & 12.365 & $12.537(-0.172)$ & - \\
\hline 403 & $3 s^{2} 3 p^{2} 4 p$ & ${ }^{2} \mathrm{~F}_{5 / 2}$ & 12.736 & $12.933(-0.197)$ & - \\
\hline 407 & $3 s^{2} 3 p^{2} 4 p$ & ${ }^{2} \mathrm{~F}_{7 / 2}$ & 12.771 & $12.972(-0.200)$ & - \\
\hline 484 & $3 s^{2} 3 p^{2} 4 d$ & ${ }^{4} \mathrm{P}_{5 / 2}$ & 13.745 & $13.953(-0.208)$ & - \\
\hline 487 & $3 s^{2} 3 p^{2} 4 d$ & ${ }^{4} \mathrm{~F}_{5 / 2}$ & 13.797 & $13.997(-0.200)$ & - \\
\hline 490 & $3 s^{2} 3 p^{2} 4 d$ & ${ }^{4} \mathrm{P}_{3 / 2}$ & 13.827 & $14.026(-0.199)$ & - \\
\hline 491 & $3 s^{2} 3 p^{2} 4 d$ & ${ }^{2} \mathrm{~F}_{5 / 2}$ & 13.815 & $14.045(-0.230)$ & - \\
\hline 503 & $3 s^{2} 3 p^{2} 4 d$ & ${ }^{2} \mathrm{~F}_{7 / 2}$ & 13.880 & $14.113(-0.233)$ & - \\
\hline 519 & $3 s^{2} 3 p^{2} 4 d$ & ${ }^{4} \mathrm{D}_{7 / 2}$ & 13.962 & $14.163(-0.201)$ & - \\
\hline 528 & $3 s^{2} 3 p^{2} 4 d$ & ${ }^{2} \mathrm{D}_{5 / 2}$ & 13.988 & $14.206(-0.218)$ & - \\
\hline 531 & $3 s^{2} 3 p^{2} 4 d$ & ${ }^{2} \mathrm{D}_{3 / 2}$ & 14.001 & $14.221(-0.220)$ & - \\
\hline 542 & $3 s^{2} 3 p^{2} 4 d$ & ${ }^{2} \mathrm{~F}_{7 / 2}$ & 14.118 & $14.333(-0.215)$ & - \\
\hline
\end{tabular}

Table 2. continued.

\begin{tabular}{lccccc}
\hline \hline$i$ & Conf. & Lev. & $E_{\text {exp }}$ & $E_{\mathrm{t}}$ & $E_{\mathrm{t}}(\mathrm{S} 05)$ \\
\hline 545 & $3 \mathrm{~s}^{2} 3 \mathrm{p}^{2} 4 \mathrm{~d}$ & ${ }^{2} \mathrm{~F}_{5 / 2}$ & 14.137 & $14.344(-0.206)$ & - \\
554 & $3 \mathrm{~s}^{2} 3 \mathrm{p}^{2} 4 \mathrm{~d}$ & ${ }^{2} \mathrm{D}_{5 / 2}$ & 14.140 & $14.388(-0.249)$ & - \\
568 & $3 \mathrm{~s}^{2} 3 \mathrm{p}^{2} 4 \mathrm{~d}$ & ${ }^{2} \mathrm{P}_{3 / 2}$ & 14.268 & $14.495(-0.227)$ & - \\
587 & $3 \mathrm{~s}^{2} 3 \mathrm{p}^{2} 4 \mathrm{~d}$ & ${ }^{2} \mathrm{~S}_{1 / 2}$ & 14.302 & $14.581(-0.279)$ & - \\
618 & $3 \mathrm{~s}^{2} 3 \mathrm{p}^{2} 4 \mathrm{f}$ & ${ }^{4} \mathrm{G}_{5 / 2}$ & 14.678 & $14.915(-0.237)$ & - \\
619 & $3 \mathrm{~s}^{2} 3 \mathrm{p}^{2} 4 \mathrm{f}$ & ${ }^{4} \mathrm{G}_{7 / 2}$ & 14.707 & $14.942(-0.235)$ & - \\
628 & $3 \mathrm{~s}^{2} 3 \mathrm{p}^{2} 4 \mathrm{f}$ & ${ }^{4} \mathrm{G}_{9 / 2}$ & 14.761 & $14.991(-0.230)$ & - \\
644 & $3 \mathrm{~s}^{2} 3 \mathrm{p}^{2} 4 \mathrm{f}$ & ${ }^{4} \mathrm{G}_{11 / 2}$ & 14.820 & $15.045(-0.225)$ & - \\
672 & $3 \mathrm{~s}^{2} 3 \mathrm{p}^{2} 4 \mathrm{f}$ & ${ }^{4} \mathrm{~F}_{7 / 2}$ & 14.866 & $15.128(-0.262)$ & - \\
688 & $3 \mathrm{~s}^{2} 3 \mathrm{p}^{2} 4 \mathrm{f}$ & ${ }^{2} \mathrm{G}_{9 / 2}$ & 14.908 & $15.193(-0.285)$ & - \\
716 & $3 \mathrm{~s}^{2} 3 \mathrm{p}^{2} 4 \mathrm{f}$ & ${ }^{2} \mathrm{H}_{11 / 2}$ & 15.192 & $15.451(-0.259)$ & - \\
719 & $3 \mathrm{~s}^{2} 3 \mathrm{p}^{2} 4 \mathrm{f}$ & ${ }^{2} \mathrm{H}_{9 / 2}$ & 15.210 & $15.469(-0.259)$ & - \\
\hline
\end{tabular}

Notes. The experimental level energies $E_{\exp }$ (in Rydbergs, from Del Zanna et al. 2004, for the $n=3$ and Fawcett et al. 1972, for $n=4$ ) are shown, together with those obtained from our scattering target $E_{\mathrm{t}}$ and those from the scattering calculation of Storey et al. (2005, S05). Values in parentheses indicate differences with $E_{\text {exp }}$. Only a selection of levels is shown.
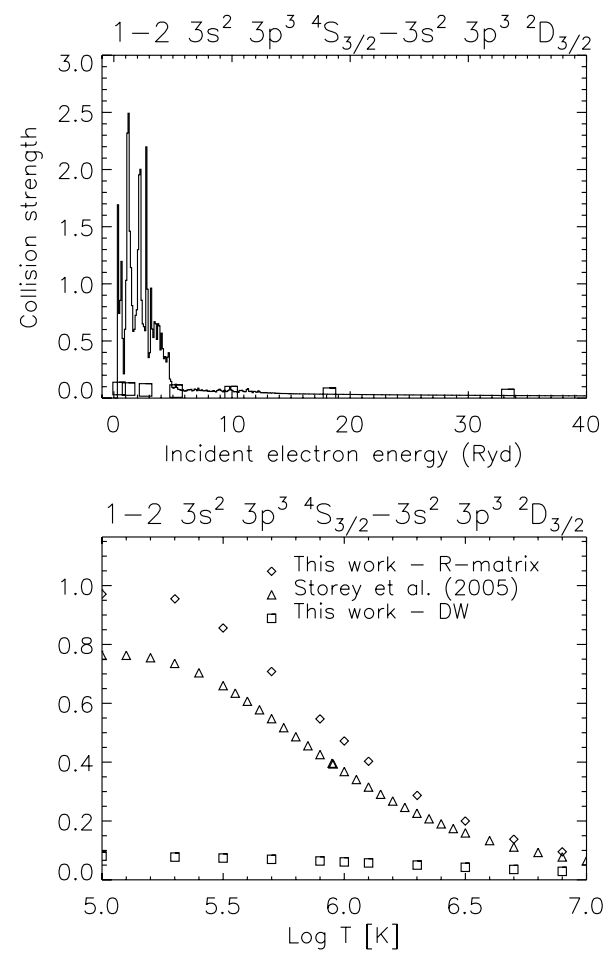

Fig. 2. Above: collision strength for the $1-2$ line, averaged over 0.1 Ryd in the resonance region. The data points are displayed in histogram mode. Boxes indicate the DW values. Below: thermally-averaged collision strengths, compared to the R-matrix results of Storey et al. (2005) and the DW ones.

diagnostics in the EUV for this ion, and are prominent in Hinode EIS spectra. We found no significant differences at densities towards the high-density limits for these transitions.

The main consequence of the present model is to lower the densities obtained from these diagnostics. This is what was needed to remove the discrepancy with the densities obtained from other ions. We have analysed various observations at low and high densities and found excellent agreement using the present atomic data. One example is provided in Fig. 9. We have considered the Hinode EIS off-limb active region spectra recorded on 2007 Aug. 19, and used in Del Zanna (2012) to 
G. Del Zanna et al.: Atomic data for astrophysics: Fe XII soft X-ray lines

Table 3. Relative intensities of the brightest lines in Fe XII in the EUV and the visible.

\begin{tabular}{|c|c|c|c|c|c|c|c|}
\hline$i-j$ & Levels & $\begin{array}{c}\text { Int } \\
\text { RM4 }\end{array}$ & $\begin{array}{c}\text { Int } \\
\text { DM05 }\end{array}$ & $\begin{array}{c}\text { Int } \\
\text { RM4+DW6 }\end{array}$ & $g f$ & $A_{j i}\left(\mathrm{~s}^{-1}\right)$ & $\lambda_{\exp }(\AA)$ \\
\hline $1-27$ & $3 s^{2} 3 p^{3}{ }^{4} S_{3 / 2}-3 s^{2} 3 p^{2} 3 d^{4} P_{5 / 2}$ & 1.0 & 1.0 & 1.0 & 3.03 & $8.7 \times 10^{10}$ & 195.119 \\
\hline $1-29$ & $3 s^{2} 3 p^{3}{ }^{4} S_{3 / 2}-3 s^{2} 3 p^{2} 3 d^{4} P_{3 / 2}$ & 0.67 & 0.67 & 0.67 & 2.03 & $8.9 \times 10^{10}$ & 193.509 \\
\hline $1-30$ & $3 s^{2} 3 p^{3}{ }^{4} S_{3 / 2}-3 s^{2} 3 p^{2} 3 d^{4} P_{1 / 2}$ & 0.31 & 0.32 & 0.31 & 1.00 & $8.8 \times 10^{10}$ & 192.394 \\
\hline $3-39$ & $3 \mathrm{~s}^{2} 3 \mathrm{p}^{3}{ }^{2} \mathrm{D}_{5 / 2}-3 \mathrm{~s}^{2} 3 \mathrm{p}^{2} 3 \mathrm{~d}^{2} \mathrm{~F}_{7 / 2}$ & $8.3 \times 10^{-2}$ & $5.3 \times 10^{-2}$ & $8.6 \times 10^{-2}$ & 4.62 & $1.1 \times 10^{11}$ & 186.887 \\
\hline $3-32$ & $3 \mathrm{~s}^{2} 3 \mathrm{p}^{3}{ }^{2} \mathrm{D}_{5 / 2}-3 \mathrm{~s}^{2} 3 \mathrm{p}^{2} 3 \mathrm{~d}^{2} \mathrm{D}_{5 / 2}$ & $4.2 \times 10^{-2}$ & $3.2 \times 10^{-2}$ & $4.3 \times 10^{-2}$ & 1.04 & $2.7 \times 10^{10}$ & 203.728 \\
\hline $3-34$ & $3 \mathrm{~s}^{2} 3 \mathrm{p}^{3}{ }^{2} \mathrm{D}_{5 / 2}-3 \mathrm{~s}^{2} 3 \mathrm{p}^{2} 3 \mathrm{~d}^{2} \mathrm{D}_{5 / 2}$ & $2.9 \times 10^{-2}$ & $1.8 \times 10^{-2}$ & $3.0 \times 10^{-2}$ & 1.58 & $4.5 \times 10^{10}$ & 196.640 \\
\hline $6-84$ & $3 \mathrm{~s} 3 \mathrm{p}^{4}{ }^{4} \mathrm{P}_{5 / 2}-3 \mathrm{~s} 3 \mathrm{p}^{3} 3 \mathrm{~d}^{4} \mathrm{D}_{7 / 2}$ & $1.9 \times 10^{-2}$ & $1.8 \times 10^{-2}$ & $2.0 \times 10^{-2}$ & 3.15 & $7.2 \times 10^{10}$ & 190.040 \\
\hline $2-36$ & $3 s^{2} 3 p^{3}{ }^{2} D_{3 / 2}-3 s^{2} 3 p^{2} 3 d^{2} F_{5 / 2}$ & $1.9 \times 10^{-2}$ & $1.0 \times 10^{-2}$ & $2.0 \times 10^{-2}$ & 3.25 & $1.0 \times 10^{11}$ & 186.854 \\
\hline $2-31$ & $3 \mathrm{~s}^{2} 3 \mathrm{p}^{3}{ }^{2} \mathrm{D}_{3 / 2}-3 \mathrm{~s}^{2} 3 \mathrm{p}^{2} 3 \mathrm{~d}^{2} \mathrm{D}_{3 / 2}$ & $1.4 \times 10^{-2}$ & $1.2 \times 10^{-2}$ & $1.5 \times 10^{-2}$ & 0.20 & $7.8 \times 10^{9}$ & 206.368 \\
\hline $1-6$ & $3 s^{2} 3 p^{3}{ }^{4} S_{3 / 2}-3 s 3 p^{4}{ }^{4} P_{5 / 2}$ & 0.37 & 0.31 & 0.38 & 0.19 & $1.6 \times 10^{9}$ & 364.467 \\
\hline $1-7$ & $3 s^{2} 3 p^{3}{ }^{4} S_{3 / 2}-3 s 3 p^{4}{ }^{4} P_{3 / 2}$ & 0.21 & 0.18 & 0.21 & 0.13 & $1.6 \times 10^{9}$ & 352.106 \\
\hline $1-8$ & $3 s^{2} 3 p^{3}{ }^{4} S_{3 / 2}-3 s 3 p^{4}{ }^{4} P_{1 / 2}$ & 0.11 & $9 \times 10^{-2}$ & 0.11 & $6.7 \times 10^{-2}$ & $1.9 \times 10^{9}$ & 348.852 \\
\hline $3-16$ & $3 s^{2} 3 p^{3}{ }^{2} D_{5 / 2}-3 s^{2} 3 p^{2} 3 d^{4} F_{7 / 2}$ & $5.5 \times 10^{-2}$ & $4 \times 10^{-2}$ & $5.8 \times 10^{-2}$ & $1.8 \times 10^{-3}$ & $2.2 \times 10^{7}$ & 256.410 \\
\hline $3-10$ & $3 \mathrm{~s}^{2} 3 \mathrm{p}^{3}{ }^{2} \mathrm{D}_{5 / 2}-3 \mathrm{~s} 3 \mathrm{p}^{4} \mathrm{C}_{5 / 2}$ & $4.1 \times 10^{-2}$ & $2.3 \times 10^{-2}$ & $4.3 \times 10^{-2}$ & 0.29 & $2.8 \times 10^{9}$ & 338.263 \\
\hline $3-20$ & $3 \mathrm{~s}^{2} 3 \mathrm{p}^{32} \mathrm{D}_{5 / 2}-3 \mathrm{~s}^{2} 3 \mathrm{p}^{2} 3 \mathrm{~d}^{4} \mathrm{D}_{7 / 2}$ & $4.0 \times 10^{-2}$ & $2.6 \times 10^{-2}$ & $4.3 \times 10^{-2}$ & $2.3 \times 10^{-3}$ & $3.0 \times 10^{7}$ & 249.388 \\
\hline $3-11$ & $3 s^{2} 3 p^{3}{ }^{2} D_{5 / 2}-3 s^{2} 3 p^{2} 3 d^{2} P_{3 / 2}$ & $3.8 \times 10^{-2}$ & $2.5 \times 10^{-2}$ & $4.0 \times 10^{-2}$ & 0.39 & $7.6 \times 10^{9}$ & 291.010 \\
\hline $6-17$ & $3 \mathrm{~s} 3 \mathrm{p}^{4}{ }^{4} \mathrm{P}_{5 / 2}-3 \mathrm{~s}^{2} 3 \mathrm{p}^{2} 3 \mathrm{~d}^{4} \mathrm{~F}_{9 / 2}$ & $3.6 \times 10^{-2}$ & $2.3 \times 10^{-2}$ & $3.9 \times 10^{-2}$ & - & 56. & 592.600 \\
\hline $1-2$ & $3 s^{2} 3 p^{3}{ }^{4} S_{3 / 2}-3 s^{2} 3 p^{3}{ }^{2} D_{3 / 2}$ & 0.45 & 0.32 & 0.46 & - & 44. & 2406.41 \\
\hline $1-3$ & $3 s^{2} 3 p^{3}{ }^{4} S_{3 / 2}-3 s^{2} 3 p^{3}{ }^{2} D_{5 / 2}$ & 0.22 & 0.19 & 0.23 & - & 1.7 & 2169.76 \\
\hline $1-5$ & $3 s^{2} 3 p^{3}{ }^{4} \mathrm{~S}_{3 / 2}-3 s^{2} 3 p^{3}{ }^{2} \mathrm{P}_{3 / 2}$ & $8.0 \times 10^{-2}$ & $5.6 \times 10^{-2}$ & $8.2 \times 10^{-2}$ & - & $3.2 \times 10^{2}$ & 1242.01 \\
\hline $2-5$ & $3 s^{2} 3 p^{3}{ }^{2} D_{3 / 2}-3 s^{2} 3 p^{3}{ }^{2} \mathrm{P}_{3 / 2}$ & $4.8 \times 10^{-2}$ & $3.8 \times 10^{-2}$ & $4.9 \times 10^{-2}$ & - & $1.9 \times 10^{2}$ & 2566.77 \\
\hline $1-4$ & $3 s^{2} 3 p^{3}{ }^{4} S_{3 / 2}-3 s^{2} 3 p^{3}{ }^{2} P_{1 / 2}$ & $4.7 \times 10^{-2}$ & $3.2 \times 10^{-2}$ & $4.9 \times 10^{-2}$ & - & $1.7 \times 10^{2}$ & 1349.40 \\
\hline $3-5$ & $3 s^{2} 3 p^{3}{ }^{2} D_{5 / 2}-3 s^{2} 3 p^{3}{ }^{2} P_{3 / 2}$ & $2.0 \times 10^{-2}$ & $1.3 \times 10^{-2}$ & $2.0 \times 10^{-2}$ & - & 78. & 2904.70 \\
\hline $2-4$ & $3 s^{2} 3 p^{3}{ }^{2} \mathrm{D}_{3 / 2}-3 s^{2} 3 p^{3}{ }^{2} \mathrm{P}_{1 / 2}$ & $1.9 \times 10^{-2}$ & $1.2 \times 10^{-2}$ & $2.0 \times 10^{-2}$ & - & 69. & 3072.06 \\
\hline
\end{tabular}

Notes. The lines are grouped in different transition arrays, and are displayed in decreasing order of intensity. Columns 3-5 show the relative line intensities (photons) Int $=N_{j} A_{j i} / N_{\mathrm{e}}$ calculated at $\log N_{\mathrm{e}}\left[\mathrm{cm}^{-3}\right]=8$ and $\log T_{\mathrm{e}}[\mathrm{K}]=6.2$ with the RM4, DM05, and RM4+DW6 ion models (see text). Intensities are normalised to the intensity of the brightest line. Columns 6, 7 show the $g f$ and $A$ values calculated in this work. The last column shows the wavelengths corresponding to the experimental energies.
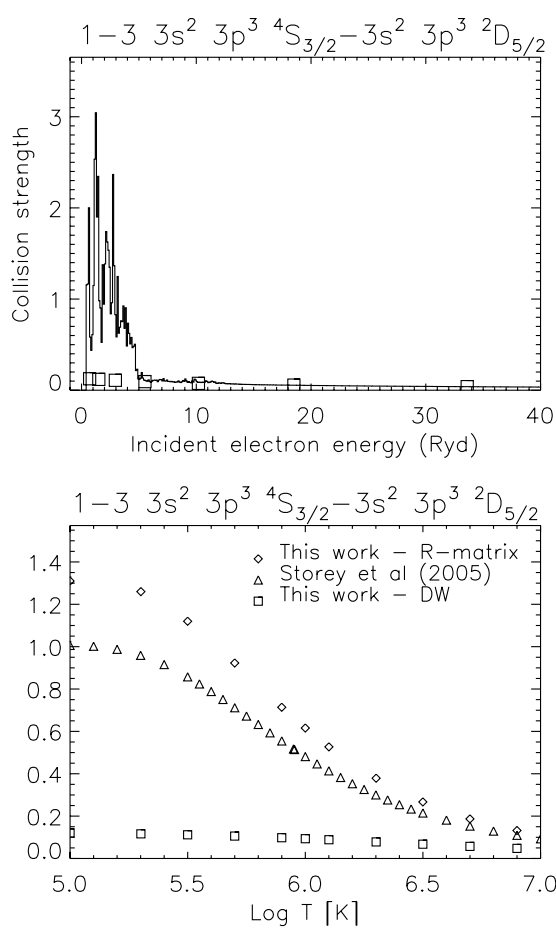

Fig. 3. Same as Fig. 2, for the 1-3 transition.

benchmark all the coronal ions. As seen in other cases, Fe XII produced higher densities compared to those from all the other ions. Figure 9 (top) shows the emissivity ratio curves obtained with the previous DM05 atomic data, indicating a density log
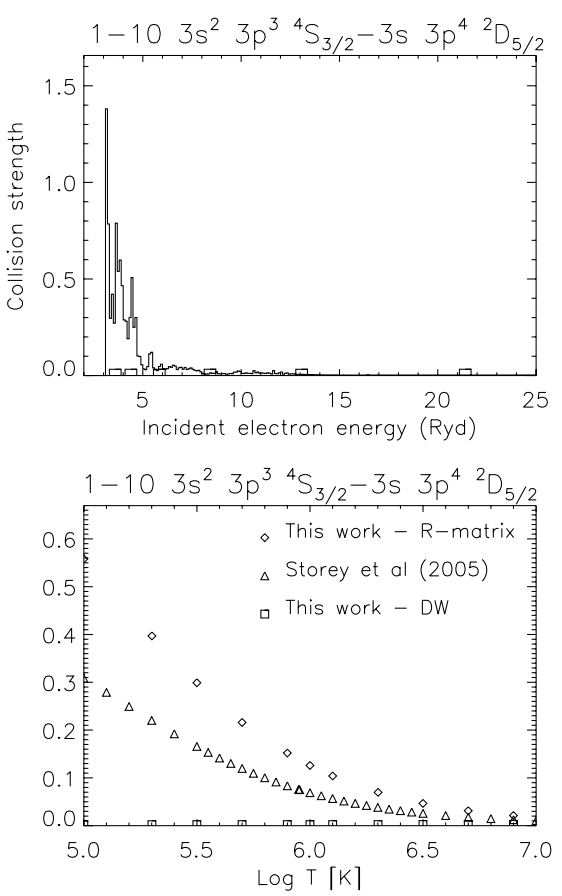

Fig. 4. Same as Fig. 2, for the 1-10 transition.

$N_{\mathrm{e}}\left[\mathrm{cm}^{-3}\right]=8.8$. These curves are obtained by dividing the observed intensities of the lines with their predicted emissivity as a function of the electron density. The crossing of the curves indicates agreement between observed and predicted line intensities 

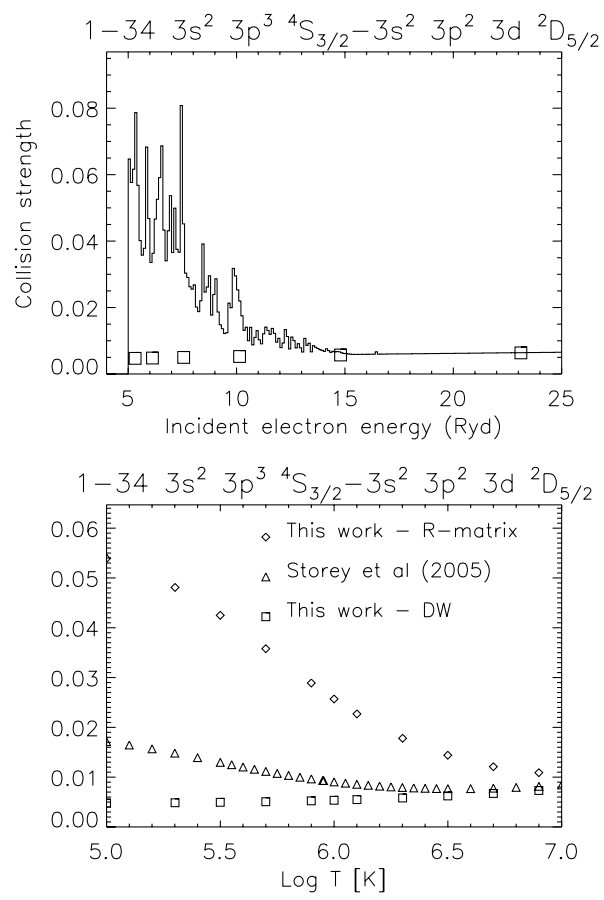

Fig. 5. Same as Fig. 2, for the 1-34 transition.
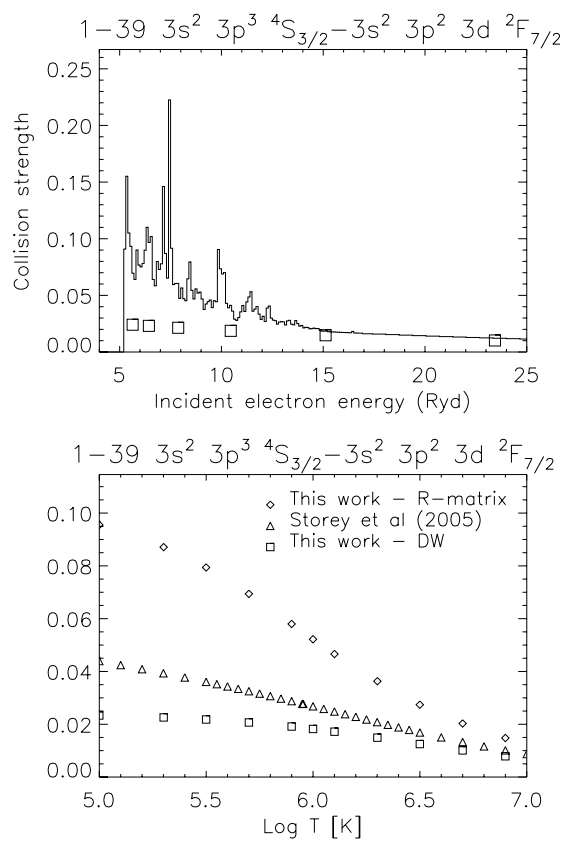

Fig. 6. Same as Fig. 2, for the 1-39 transition.

at $\log N_{\mathrm{e}}\left[\mathrm{cm}^{-3}\right]=8.8$ (for further details on this emissivity ratio technique see Del Zanna et al. 2004). The measurements from other ions emitted at similar temperatures such as $\mathrm{Si}$ x indicated a density $\log N_{\mathrm{e}}\left[\mathrm{cm}^{-3}\right]=8.5$. Figure 9 (bottom) shows the emissivity ratio curves obtained with the current RM4+DW6 model (an extension of RM4, as discussed below), indicating a density $\log N_{\mathrm{e}}\left[\mathrm{cm}^{-3}\right]=8.5$, in excellent agreement with the $\mathrm{Si}$ x results.

One question then naturally arises: what causes these higher intensities at low coronal densities? The differences with the previous DM05 model turn out to be due to a combination of subtle effects, mostly due to small increases in the excitations of vari-
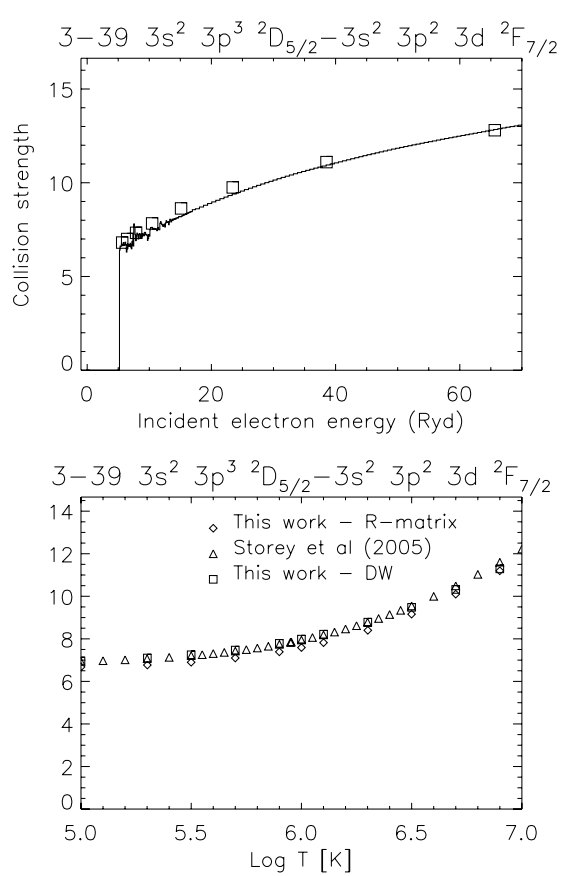

Fig. 7. Same as Fig. 2, for the 3-39 transition.
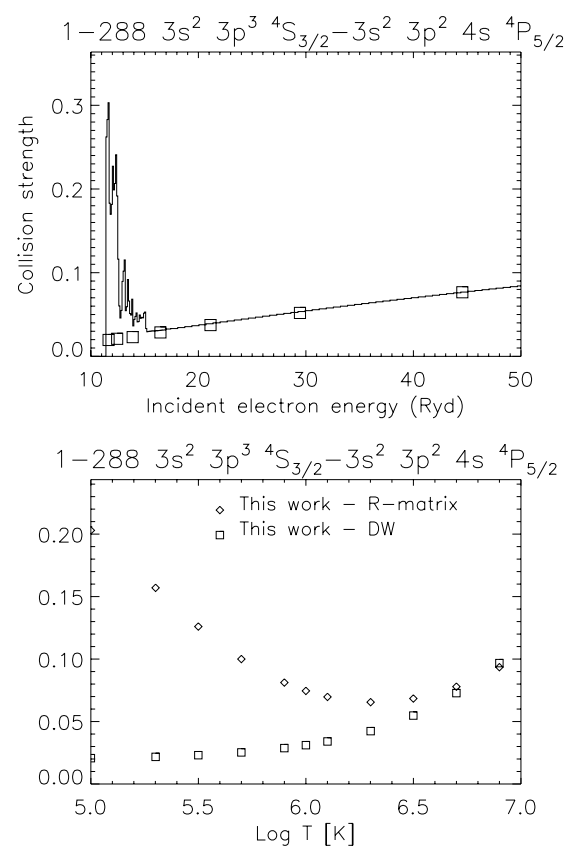

Fig. 8. Same as Fig. 2, for the 1-288 transition, the strongest soft X-ray line among those identified by Fawcett et al. (1972). Note the strong enhancement due to the resonances.

ous levels due to the extra resonances we obtain with this much larger scattering calculation.

In order to make sure that no other effects are in play, we performed a full new R-matrix calculation where we adopted the same target basis and orbital scaling parameters as in RM4, but included this time only the lowest 58 terms and 143 levels as was done in Storey et al. (2005). In order to match the energy resolution of the RM4 calculation, we calculated collision strengths for 4200 points in the resonance region. We refer to this 58-terms calculation as RM4_58T. Figure 10 shows a compari- 
Table 4. Populations of a few Fe XII $(n=3)$ key levels.

\begin{tabular}{|c|c|c|c|c|c|c|c|}
\hline$i$ & Lev. & $\begin{array}{c}\text { POP(\%) } \\
\text { DM05 }\end{array}$ & $\begin{array}{l}\mathrm{RD}(\%) \\
\mathrm{DM} 05\end{array}$ & $\begin{array}{c}\mathrm{EXC}(\%) \\
\text { DM05 }\end{array}$ & $\begin{array}{c}\text { POP(\%) } \\
\text { RM4 }\end{array}$ & $\begin{array}{c}\mathrm{RD}(\%) \\
\mathrm{RM} 4\end{array}$ & $\begin{array}{c}\mathrm{EXC}(\%) \\
\mathrm{RM} 4\end{array}$ \\
\hline 1 & $3 s^{2} 3 p^{3}{ }^{4} S_{3 / 2}$ & 94.98 & & & 92.1 & & \\
\hline 2 & $3 s^{2} 3 p^{32} D_{3 / 2}$ & 0.32 & $\begin{array}{c}72.8 \\
22(3) \\
9(5)\end{array}$ & $22(1)$ & $\begin{array}{c}0.57 \\
24(3) \\
10(5)\end{array}$ & 76 & $19(1)$ \\
\hline 3 & $3 s^{2} 3 p^{32} D_{5 / 2}$ & 4.7 & $\begin{array}{c}77 \\
12(39) \\
9(16) \\
7(32) \\
6(19) \\
6(11)\end{array}$ & $21(1)$ & 7.2 & $\begin{array}{c}80 \\
13(39) \\
8(16) \\
6(32) \\
6(10) \\
6(20)\end{array}$ & $18(1)$ \\
\hline 34 & $3 s^{2} 3 p^{2} 3 d^{2} D_{5 / 2}$ & $2.4 \times 10^{-11}$ & 1.6 & $\begin{array}{c}98 \\
91(3) \\
7(1)\end{array}$ & $3.7 \times 10^{-11}$ & 6.9 & $\begin{array}{c}93 \\
83(3) \\
10(1)\end{array}$ \\
\hline 39 & $3 s^{2} 3 p^{2} 3 d^{2} F_{7 / 2}$ & $2.8 \times 10^{-11}$ & 0.6 & $\begin{array}{c}99 \\
92(3) \\
7(1)\end{array}$ & $4.4 \times 10^{-11}$ & 4.8 & $\begin{array}{c}95 \\
87(3) \\
8(1)\end{array}$ \\
\hline
\end{tabular}

Notes. The relative populations POP are given as percentages. RD indicates how much of the population is due to radiative decay from higher levels. EXC indicates how much of the population is due to excitation from lower levels. Some fractional contributions are noted (level numbers $i$ in parentheses). DM05 indicates values calculated with the previous ion model, RM4 with the present RM4 ion model. In both cases, the values are calculated at $\log N_{\mathrm{e}}\left[\mathrm{cm}^{-3}\right]=8$ and $\log T_{\mathrm{e}}[\mathrm{K}]=6.2$.
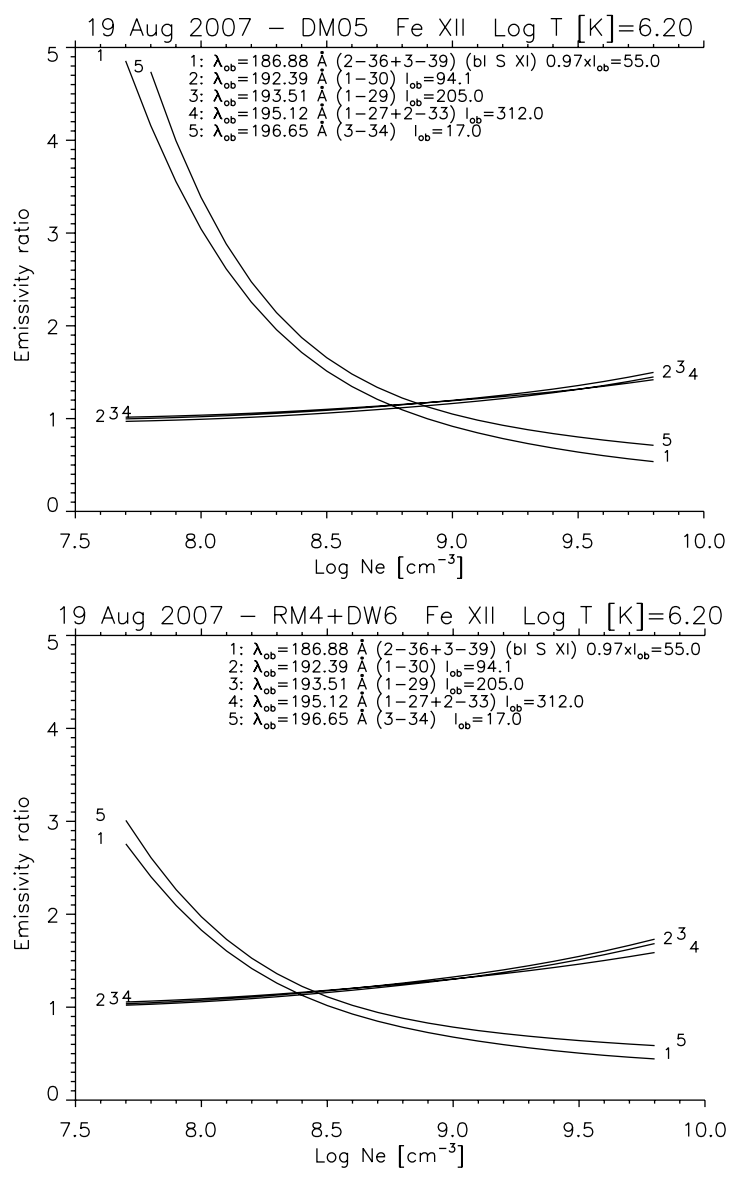

Fig. 9. Emissivity ratio curves relative to the main Fe xII EUV transitions observed by Hinode EIS on 2007 Aug. 19, using the DM05 atomic model (above) and the present RM4+DW6 (below). The wavelengths and indices of the transitions are given, as well as the observed intensity $I_{\mathrm{ob}}$ (see Del Zanna 2012, for details).

son between the collision strengths of a few key transitions for the RM4, the RM4_58T, and the Storey et al. (2005) R-matrix
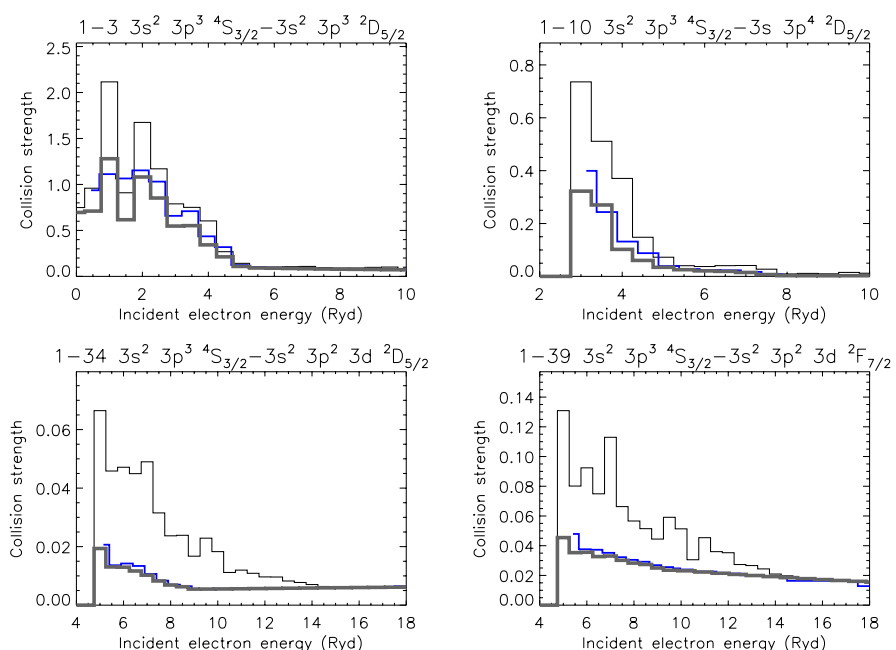

Fig. 10. Collision strengths from the RM4 calculation for a selection of transitions, averaged over 0.5 Ryd in the resonance region (displayed in histogram mode, thin black lines). The thick (blue) lines indicate the Storey et al. (2005) values, and the thicker (grey) lines those from the RM4_58T calculation.

calculations. Overall agreement between the RM4_58T and the Storey et al. (2005) results is found for all transitions, however the RM4 present some clear enhancements. Table 4 provides a summary of the populations of some of the key levels, as calculated with the previous (DM05) and current (RM4) ion models at quiet-Sun densities $\left(\log N_{\mathrm{e}}\left[\mathrm{cm}^{-3}\right]=8\right)$.

The increase in the intensity of the 3-39 $186.88 \AA$ transition is due to an increased population for level 39, which in turn is mainly due to two effects, the excitation from level 3 (by 87\%) and that one from the ground state $(8 \%)$. The first is enhanced due to an increased population of level 3 from $4.7 \%$ to $7.2 \%$ (i.e. by $50 \%$ ). In turn, level 3 becomes populated directly from the ground state by $18 \%$ and by $80 \%$ via cascading from a range of $n=3$ levels. The direct excitation from the ground state is increased as shown in Fig. 10. Cascading for level 3 is also in- 
Table 5. Relative intensities of the brightest Fe XII lines in in the soft X-rays.

\begin{tabular}{|c|c|c|c|c|c|c|c|c|c|}
\hline$i-j$ & Levels & $\begin{array}{c}\text { Int } \\
1.0 \times 10^{8} \\
\text { DW4 }\end{array}$ & $\begin{array}{c}\text { Int } \\
1.0 \times 10^{8} \\
\mathrm{RM} 4\end{array}$ & $\begin{array}{c}\text { Int } \\
1.0 \times 10^{8} \\
\text { RM4+DW6 }\end{array}$ & $\begin{array}{c}\text { Int } \\
1.0 \times 10^{12} \\
\mathrm{RM} 4\end{array}$ & $g f$ & $A_{j i}\left(\mathrm{~s}^{-1}\right)$ & $\lambda_{\exp }(\AA)$ & $\lambda_{\text {th }}(\AA)$ \\
\hline $6-467$ & $3 \mathrm{~s} 3 \mathrm{p}^{4}{ }^{4} \mathrm{P}_{5 / 2}-3 \mathrm{~s} 3 \mathrm{p}^{3} 4 \mathrm{~s}^{4} \mathrm{~S}_{3 / 2}$ & $1.4 \times 10^{-2}$ & $1.5 \times 10^{-2}$ & $1.5 \times 10^{-2}$ & $4.8 \times 10^{-3}$ & 0.33 & $8.0 \times 10^{10}$ & - & 80.76 \\
\hline $7-467$ & $3 \mathrm{~s} 3 p^{4}{ }^{4} P_{3 / 2}-3 s 3 p^{3} 4 s^{4} S_{3 / 2}$ & $8.3 \times 10^{-3}$ & $8.5 \times 10^{-3}$ & $8.5 \times 10^{-3}$ & $2.8 \times 10^{-3}$ & 0.19 & $4.6 \times 10^{10}$ & - & 81.39 \\
\hline $6-390$ & $3 s 3 p^{4}{ }^{4} P_{5 / 2}-3 s^{2} 3 p^{2} 4 p^{4} S_{3 / 2}$ & $7.9 \times 10^{-3}$ & $8.1 \times 10^{-3}$ & $8.1 \times 10^{-3}$ & $2.7 \times 10^{-3}$ & $6.9 \times 10^{-2}$ & $1.4 \times 10^{10}$ & - & 89.03 \\
\hline $1-288$ & $3 s^{2} 3 p^{3}{ }^{4} S_{3 / 2}-3 s^{2} 3 p^{2} 4 s^{4} P_{5 / 2}$ & $4.7 \times 10^{-3}$ & $7.3 \times 10^{-3}$ & $8.0 \times 10^{-3}$ & $3.4 \times 10^{-3}$ & 0.29 & $5.0 \times 10^{10}$ & 79.488 & $78.29(-1.2)$ \\
\hline $7-390$ & $3 s 3 p^{4}{ }^{4} P_{3 / 2}-3 s^{2} 3 p^{2} 4 p^{4} S_{3 / 2}$ & $6.8 \times 10^{-3}$ & $7.0 \times 10^{-3}$ & $7.1 \times 10^{-3}$ & $2.4 \times 10^{-3}$ & $6.0 \times 10^{-2}$ & $1.2 \times 10^{10}$ & - & 89.78 \\
\hline $1-278$ & $3 s^{2} 3 p^{3}{ }^{4} S_{3 / 2}-3 s^{2} 3 p^{2} 4 s^{4} P_{3 / 2}$ & $2.9 \times 10^{-3}$ & $4.5 \times 10^{-3}$ & $4.7 \times 10^{-3}$ & $2.1 \times 10^{-3}$ & 0.20 & $5.1 \times 10^{10}$ & 80.022 & $78.78(-1.2)$ \\
\hline $8-467$ & $3 \mathrm{~s} 3 \mathrm{p}^{4}{ }^{4} \mathrm{P}_{1 / 2}-3 \mathrm{~s} 3 \mathrm{p}^{3} 4 \mathrm{~s}^{4} \mathrm{~S}_{3 / 2}$ & $4.2 \times 10^{-3}$ & $4.3 \times 10^{-3}$ & $4.3 \times 10^{-3}$ & $1.4 \times 10^{-3}$ & $9.9 \times 10^{-2}$ & $2.3 \times 10^{10}$ & - & 81.67 \\
\hline $8-390$ & $3 s 3 p^{4}{ }^{4} P_{1 / 2}-3 s^{2} 3 p^{2} 4 p^{4} S_{3 / 2}$ & $4.0 \times 10^{-3}$ & $4.1 \times 10^{-3}$ & $4.1 \times 10^{-3}$ & $1.4 \times 10^{-3}$ & $3.6 \times 10^{-2}$ & $6.9 \times 10^{9}$ & - & 90.13 \\
\hline 29-390 & $3 s^{2} 3 p^{2} 3 d^{4} P_{3 / 2}-3 s^{2} 3 p^{2} 4 p{ }^{4} S_{3 / 2}$ & $3.7 \times 10^{-3}$ & $3.8 \times 10^{-3}$ & $3.8 \times 10^{-3}$ & $1.3 \times 10^{-3}$ & $5.3 \times 10^{-2}$ & $6.4 \times 10^{9}$ & - & 114.90 \\
\hline $27-390$ & $3 s^{2} 3 p^{2} 3 d^{4} P_{5 / 2}-3 s^{2} 3 p^{2} 4 p{ }^{4} S_{3 / 2}$ & $3.6 \times 10^{-3}$ & $3.7 \times 10^{-3}$ & $3.7 \times 10^{-3}$ & $1.3 \times 10^{-3}$ & $5.1 \times 10^{-2}$ & $6.2 \times 10^{9}$ & - & 114.37 \\
\hline $1-484$ & $3 s^{2} 3 p^{3}{ }^{4} S_{3 / 2}-3 s^{2} 3 p^{2} 4 d^{4} P_{5 / 2}$ & $2.6 \times 10^{-3}$ & $3.2 \times 10^{-3}$ & $3.8 \times 10^{-3}$ & $1.3 \times 10^{-3}$ & 0.52 & $1.3 \times 10^{11}$ & 66.297 & $65.31(-1.0)$ \\
\hline $17-383$ & $3 s^{2} 3 p^{2} 3 d^{4} F_{9 / 2}-3 s^{2} 3 p^{2} 4 p^{4} D_{7 / 2}$ & $2.6 \times 10^{-3}$ & $3.0 \times 10^{-3}$ & $3.4 \times 10^{-3}$ & $2.1 \times 10^{-3}$ & 0.46 & $3.2 \times 10^{10}$ & - & 107.04 \\
\hline $1-487$ & $3 s^{2} 3 p^{3}{ }^{4} S_{3 / 2}-3 s^{2} 3 p^{2} 4 d^{4} F_{5 / 2}$ & $2.0 \times 10^{-3}$ & $2.5 \times 10^{-3}$ & $3.3 \times 10^{-3}$ & $1.0 \times 10^{-3}$ & 0.38 & $9.6 \times 10^{10}$ & 66.047 & $65.10(-0.9)$ \\
\hline $1-272$ & $3 s^{2} 3 p^{3}{ }^{4} S_{3 / 2}-3 s^{2} 3 p^{2} 4 s^{4} P_{1 / 2}$ & $1.5 \times 10^{-3}$ & $2.2 \times 10^{-3}$ & $2.3 \times 10^{-3}$ & $1.0 \times 10^{-3}$ & 0.10 & $5.2 \times 10^{10}$ & 80.515 & $79.20(-1.3)$ \\
\hline $1-490$ & $3 s^{2} 3 p^{3}{ }^{4} S_{3 / 2}-3 s^{2} 3 p^{2} 4 d^{4} P_{3 / 2}$ & $1.7 \times 10^{-3}$ & $2.2 \times 10^{-3}$ & $2.7 \times 10^{-3}$ & $9.1 \times 10^{-4}$ & 0.41 & $1.5 \times 10^{11}$ & 65.905 & $64.97(-0.9)$ \\
\hline $16-370$ & $3 s^{2} 3 p^{2} 3 d^{4} F_{7 / 2}-3 s^{2} 3 p^{2} 4 p^{4} D_{5 / 2}$ & $1.8 \times 10^{-3}$ & $2.1 \times 10^{-3}$ & $2.3 \times 10^{-3}$ & $1.2 \times 10^{-3}$ & 0.34 & $3.2 \times 10^{10}$ & 108.605 & $107.16(-1.4)$ \\
\hline $30-390$ & $3 s^{2} 3 p^{2} 3 d^{4} P_{1 / 2}-3 s^{2} 3 p^{2} 4 p^{4} S_{3 / 2}$ & $2.0 \times 10^{-3}$ & $2.1 \times 10^{-3}$ & $2.1 \times 10^{-3}$ & $7.1 \times 10^{-4}$ & $2.9 \times 10^{-2}$ & $3.5 \times 10^{9}$ & - & 115.25 \\
\hline $27-467$ & $3 s^{2} 3 p^{2} 3 d^{4} P_{5 / 2}-3 s 3 p^{3} 4 s^{4} S_{3 / 2}$ & $2.0 \times 10^{-3}$ & $2.0 \times 10^{-3}$ & $2.0 \times 10^{-3}$ & $6.7 \times 10^{-4}$ & $7.1 \times 10^{-2}$ & $1.1 \times 10^{10}$ & - & 101.09 \\
\hline 30-666 & $3 s^{2} 3 p^{2} 3 d^{4} P_{1 / 2}-3 s^{2} 3 p^{2} 4 f^{4} D_{1 / 2}$ & $1.8 \times 10^{-3}$ & $1.9 \times 10^{-3}$ & $1.9 \times 10^{-3}$ & $6.5 \times 10^{-4}$ & 0.70 & $2.9 \times 10^{11}$ & - & 88.58 \\
\hline $15-619$ & $3 s^{2} 3 p^{2} 3 d^{4} F_{5 / 2}-3 s^{2} 3 p^{2} 4 f^{4} G_{7 / 2}$ & $1.6 \times 10^{-3}$ & $1.7 \times 10^{-3}$ & $1.9 \times 10^{-3}$ & $8.3 \times 10^{-4}$ & 3.78 & $4.3 \times 10^{11}$ & 84.520 & $83.15(-1.4)$ \\
\hline $15-364$ & $3 s^{2} 3 p^{2} 3 d^{4} F_{5 / 2}-3 s^{2} 3 p^{2} 4 p^{4} D_{3 / 2}$ & $1.3 \times 10^{-3}$ & $1.7 \times 10^{-3}$ & $1.9 \times 10^{-3}$ & $8.6 \times 10^{-4}$ & 0.23 & $3.2 \times 10^{10}$ & 108.862 & $107.33(-1.5)$ \\
\hline $1-590$ & $3 s^{2} 3 p^{3}{ }^{4} S_{3 / 2}-3 s 3 p^{3} 4 p^{4} P_{5 / 2}$ & $1.2 \times 10^{-3}$ & $1.4 \times 10^{-3}$ & $1.7 \times 10^{-3}$ & $4.7 \times 10^{-4}$ & 0.39 & $1.1 \times 10^{11}$ & - & 62.40 \\
\hline
\end{tabular}

Notes. The lines are displayed in decreasing order of intensity (photons) Int $=N_{j} A_{j i} / N_{\mathrm{e}}$ relative to the strongest 1-27 line (in the EUV). The intensities were calculated at $\log T_{\mathrm{e}}[\mathrm{K}]=6.2$. Columns 3-5 show the relative intensities calculated at $\log N_{\mathrm{e}}\left[\mathrm{cm}^{-3}\right]=8$ with the DW4, RM4, and RM4+DW6 ion models. Column 6 shows the values at $\log N_{\mathrm{e}}\left[\mathrm{cm}^{-3}\right]=12$ from the RM4 ion model. Columns 7, 8 show the $g f$ and $A$ values calculated in this work. The last two columns show the wavelengths corresponding to the experimental and target energies. Values in parenthesis list the corresponding wavelength difference.

creased because of increases in the populations of the upper levels (e.g. levels 10), due to the extra resonances in our RM4 model (see Fig. 10). The second excitation for level 39 (from the ground state) is also increased as shown in Fig. 10.

Similar effects occur for the other density diagnostic, the 3-34 196.65 ̊ line, as shown in Table 4 and Fig. 10. Level 34 is mainly populated via excitation from level 3 (by 83\%) and from the ground state (10\%), and both excitations are enhanced in the present model.

\subsection{The soft $X$-ray lines}

To look at the effects of resonances, we have built two ion models for the $n=4$ levels. One with the R-matrix $n=4$ rates (RM4), and one with the DW rates (DW4). In both cases, the same set of $A$-values (obtained with experimental and best energies) were used. The relative intensities of the brightest Fe xII soft X-rays lines are shown in Table 5. The lines that turn out to be most affected by resonance enhancement are those within the $3 s^{2} 3 p^{2} 4 s$ and $3 s^{2} 3 p^{2} 4 d$ configurations, where increases of about $50 \%$ are found, i.e. not as much as we found for the Fe $\mathrm{x}$ case, but still significant.

We looked at the populations of some $3 \mathrm{~s}^{2} 3 \mathrm{p}^{2} 4 l$ levels, and found that the $3 \mathrm{~s}^{2} 3 \mathrm{p}^{2} 4 \mathrm{~s}$ are affected by about $30 \%$ by cascading from higher levels, while for the others the main excitation is directly from the ground state.

As in the Fex case, both the DW and R-matrix calculations indicate that a number of strong transitions are unidentified, in particular those from the $3 \mathrm{~s} 3 \mathrm{p}^{3} 4 \mathrm{~s}$ and $3 \mathrm{~s}^{2} 3 \mathrm{p}^{2} 4 \mathrm{p}$ levels.
Indeed, at coronal densities, many of the strongest transitions are unidentified.

\subsection{DW calculations for the $n=5,6$ levels and cascading effects}

To estimate the effects of further cascading from even higher levels, we built a new target by adding the following configurations to the R-matrix $n=4$ one (RM4): $3 \mathrm{~s}^{2} 3 \mathrm{p}^{2} 5 l(l=\mathrm{s}, \mathrm{p}, \mathrm{d}, \mathrm{f}, \mathrm{g})$, $3 \mathrm{~s} 3 \mathrm{p}^{3} 5 l(l=\mathrm{s}, \mathrm{p}, \mathrm{d}, \mathrm{f}, \mathrm{g}), 3 \mathrm{p}^{4} 5 l(l=\mathrm{s}, \mathrm{p}, \mathrm{d}, \mathrm{f}, \mathrm{g}), 3 \mathrm{~s}^{2} 3 \mathrm{p}^{2} 6 l$ $(l=\mathrm{s}, \mathrm{p}, \mathrm{d}, \mathrm{f}, \mathrm{g}), 3 \mathrm{~s} 3 \mathrm{p}^{3} 6 l(l=\mathrm{s}, \mathrm{p}, \mathrm{d}, \mathrm{f}, \mathrm{g}), 3 \mathrm{p}^{4} 6 l(l=\mathrm{s}, \mathrm{p}, \mathrm{d}, \mathrm{f}, \mathrm{g})$. We kept the scaling parameters for the $n=4$ the same, and obtained those for the $n=5,6$, which are listed in Table 1 . This was done to try and keep similar energies (and ordering of the levels) for the $n=4$ levels. The total target is very large. It comprises 66 configurations, 1970 LS terms and 5143 fine-structure levels. We then used the DW code to calculate the excitation rates up to all of these levels, but just from the lowest 41 arising from the $3 s^{2} 3 p^{3}, 3 s 3 p^{4}$, and $3 s^{2} 3 p^{2} 3 d$ configurations. This enables us to treat all possible metastable levels populating the upper ones.

We then calculated separately the radiative rates between all the 5143 levels, and matched the ordering of this calculation with that of the 912-levels RM4. We then merged the rates and $A$-values from RM4 with those from this DW run, and built an ion model, which we indicate as RM4+DW6. The relative intensities with the RM4+DW6 model of the main soft X-ray lines are shown in Table 5 while those for the EUV lines are listed in Table 3.

Some transitions from the $3 s^{2} 3 p^{2} 4 s$ and $3 s^{2} 3 p^{2} 4 p$ configurations are increased by about $10 \%$. The decays from the 
Table 6. Main cascading affecting the populations of two Fe XII $(n=4)$ levels.

\begin{tabular}{|c|c|c|c|c|c|c|c|}
\hline$l$ & Lower Lev. & $u$ & Upper Lev. & RM4 & RM4+DW5 & RM4+DW6 & \\
\hline 288 & $3 s^{2} 3 p^{2} 4 s^{4} P_{5 / 2}$ & $\begin{array}{c}703 \\
390 \\
1124 \\
2049 \\
383 \\
1116 \\
2053\end{array}$ & $\begin{array}{l}3 s 3 p^{3} 4 s^{4} S_{3 / 2} \\
3 s 3 p^{3} 4 p^{4} S_{3 / 2} \\
3 s ~ 3 p^{3} 5 p^{4} S_{3 / 2} \\
3 s ~ 3 p^{3} 6 p^{4} S_{3 / 2} \\
\text { 3s } 3 p^{3} 4 p^{4} D_{7 / 2} \\
3 s ~ 3 p^{3} 5 p^{4} D_{7 / 2} \\
\text { 3s } 3 p^{3} 6 p^{4} D_{7 / 2}\end{array}$ & $\begin{array}{c}8.28 \\
29.9 \\
18.8 \\
7.7 \\
- \\
- \\
1.2 \\
- \\
-\end{array}$ & $\begin{array}{c}8.90 \\
34.9 \\
17.6 \\
7.2 \\
5.8 \\
- \\
1.2 \\
0.5 \\
-\end{array}$ & $\begin{array}{c}9.13 \\
36.5 \\
17.1 \\
7.0 \\
5.7 \\
2.0 \\
1.2 \\
0.5 \\
0.1\end{array}$ & $\begin{array}{c}\mathrm{POP}(\%) \\
\mathrm{RD}(\%)\end{array}$ \\
\hline 487 & $3 s^{2} 3 p^{2} 4 d^{4} F_{5 / 2}$ & $\begin{array}{l}1092 \\
1458 \\
2305 \\
\end{array}$ & $\begin{array}{l}3 s^{2} 3 p^{2} 5 p^{4} P_{3 / 2} \\
3 s^{2} 3 p^{2} 5 f^{4} D_{7 / 2} \\
3 s^{2} 3 p^{2} 6 f{ }^{4} D_{7 / 2}\end{array}$ & $\begin{array}{c}1.51 \\
0.2 \\
- \\
- \\
-\end{array}$ & $\begin{array}{c}1.95 \\
22.4 \\
9.6 \\
2.5 \\
-\end{array}$ & $\begin{array}{c}1.96 \\
23.1 \\
9.6 \\
2.5 \\
0.6\end{array}$ & $\begin{array}{c}\mathrm{POP}(\%) \\
\mathrm{RD}(\%)\end{array}$ \\
\hline
\end{tabular}

Notes. The relative populations POP (in percentage) of the levels are in units of $10^{-12}$. RD indicates how much of the population (in percentage) is due to radiative decay from higher levels. Some of the main cascading levels are shown, with their percentage contribution to the population of the lower level. The values are calculated at $\log N_{\mathrm{e}}\left[\mathrm{cm}^{-3}\right]=8$ and $\log T_{\mathrm{e}}[\mathrm{K}]=6.2$.

$3 s^{2} 3 p^{2} 4 d$ levels are increased by up to about $30 \%$. Some increases for the $3 \mathrm{~d}$ transitions due to cascading effects are also present, but are normally smaller, of the order of $5 \%$.

One interesting issue is whether the RM4+DW6 model includes all the main cascading processes for the $n=4$ levels, and how much the contribution of further cascading from $n \geq 7$ levels would be. In our case, we expect further cascading from levels with $n \geq 7$ to be small, of the order of a few percent at most. The cascading obviously becomes smaller with increasing principal quantum number $n$ since the direct excitations from the ground state (hence the populations) decrease as $n^{-3}$. However, an exact calculation is non-trivial, requiring a proper scattering and structure calculation, and the building of a very large ion model. Such a calculation is left to a future paper, considering that the corrections would be of the same order (or smaller) as those arising from the resonance enhancements due to the $n \geq 5$ levels, not included in the R-matrix calculation.

For Fe x, Malinovsky et al. (1980) performed some approximate calculations to estimate the contributions by cascading to the $3 s^{2} 3 p^{4} 4 s$ levels. They assumed that the main cascading to the $3 \mathrm{~s}^{2} 3 \mathrm{p}^{4} 4 \mathrm{~s}$ levels would come from the $3 \mathrm{~s}^{2} 3 \mathrm{p}^{4} n \mathrm{p}$ levels. They then estimated approximate values for the excitation from the ground state assuming that they vary as $\left(n-\mu_{\mathrm{nL}}\right)^{-3}$, where $\mu_{\mathrm{nL}}$ is the quantum defect of the $n L$ orbital. They also assumed that the branching ratios to be constant and equal to the values for $n=5$. They found that cascading from the $3 \mathrm{~s}^{2} 3 \mathrm{p}^{4} 5 \mathrm{p}$ increased the intensities of the $3 s^{2} 3 p^{4} 4 s$ levels by at most $16 \%$. Cascading from the $3 \mathrm{~s}^{2} 3 \mathrm{p}^{4} n \mathrm{p}(n \geq 6)$ added at most a further $10 \%$.

We now look at two of the transitions most affected (see Table 5) by cascading in FexII, and show how complex cascading actually is. We consider three ion models, the RM4 and RM4+DW6, and an intermediate (RM4+DW5) where we have only retained the $n \leq 5$ levels. Details are given in Table 6 .

We consider first the 1-288 79.488 $\AA$ transition, the main decay from the $3 \mathrm{~s}^{2} 3 \mathrm{p}^{2} 4 \mathrm{~s}^{4} \mathrm{P}_{5 / 2}$ level. We find that adding $n=5$ levels increases the population by $7 \%$, while adding the $n=6$ ones adds only a further $2.6 \%$ overall. Looking in detail at which levels contribute most, we find that by far the dominant cascading comes from a $3 \mathrm{~s} 3 \mathrm{p}^{3} 4 \mathrm{~s}{ }^{4} \mathrm{~S}_{3 / 2}$ level (17\%), and not from the $3 \mathrm{~s}^{2} 3 \mathrm{p}^{2} n \mathrm{p}$ ones as one would have expected. This occurs because the $3 \mathrm{~s} 3 \mathrm{p}^{3} 4 \mathrm{~s}^{4} \mathrm{~S}_{3 / 2}$ level has a strong forbidden excitation rate from the ground state $(0.45$ at $1.26 \mathrm{MK})$ and a strong allowed decay $\left(A=3 \times 10^{10} \mathrm{~s}^{-1}\right)$ to the $3 \mathrm{~s}^{2} 3 \mathrm{p}^{2} 4 \mathrm{~s}^{4} \mathrm{P}_{5 / 2}$ (288) level, with a branching ratio of 0.3 . The next level of the Rydberg series ( $3 \mathrm{~s} 3 \mathrm{p}^{3} 5 \mathrm{~s}^{4} \mathrm{~S}_{3 / 2}$ ) has negligible contribution, having a small excitation rate $(0.007$ at $1.26 \mathrm{MK})$ from the ground state and a small branching ratio (0.0002) to level 288. The $3 \mathrm{~s}^{2}$ $3 \mathrm{p}^{2} n \mathrm{p}{ }^{4} \mathrm{~S}_{3 / 2}$ levels contribute $7.0,5.7$, and $2.0 \%$ respectively, however their excitation rates from the ground state $(0.4,0.08$, 0.04 at $1.26 \mathrm{MK})$ and branching ratios $(0.02,0.12,0.07)$ do not have a simple behavior. Further smaller contributions come from the $3 \mathrm{~s}^{2} 3 \mathrm{p}^{2} n \mathrm{p}{ }^{4} \mathrm{D}_{7 / 2}$ levels.

The situation is quite different for the higher $3 s^{2} 3 p^{2} 4 d^{4} F_{5 / 2}$ (487) level. The $n=4$ levels produce negligible cascading on its population. Adding $n=5$ levels increases significantly (29\%) its population. The $n=6$ levels, on the other hand, do not produce any significant cascading effects. The main cascading $(9.6 \%)$ comes from a $3 s^{2} 3 p^{2} 5 p^{4} \mathrm{P}_{3 / 2}$ level. Cascading from the $3 s^{2} 3 p^{2}$ $6{ }^{4} \mathrm{P}_{3 / 2}$ is negligible $(\leq 0.01 \%)$. Further cascading comes from the $3 \mathrm{~s}^{2} 3 \mathrm{p}^{2} 5 \mathrm{f}^{4} \mathrm{D}_{7 / 2}$ and a large number of other $n=5$ levels.

\section{Summary and conclusions}

We have presented the first R-matrix calculations for the $n=$ 4 levels in Fe XII. We have found the same issues we discovered in Fe x. Given their small collision strengths for excitations from the ground, the $3 s^{2} 3 p^{2} 4 s$ levels are mainly affected in two ways. First, the rates are increased significantly by resonances which are attached mainly to the $3 s^{2} 3 p^{2} 4 p$ levels. Second, the population of these levels is enhanced by cascading from higher levels. These two effects are not as large as in Fe x, but are nevertheless significant.

We found that a large number of strong transitions are unidentified, as we saw in other ions, in particular the decays from the $3 \mathrm{~s} 3 \mathrm{p}^{3} 4 \mathrm{~s}$ levels. The identifications of these levels will be discussed in a separate paper.

The somewhat surprising result of these new calculations is the enhancement in the populations of some important levels producing the best electron density diagnostics in the EUV for this ion. We find previous discrepancies to be resolved. The reasons for the enhancements are subtle and show how difficult it is to obtain a correct atomic model for complex ions such as Fe XII. 
Acknowledgements. G.D.Z. acknowledges the support from STFC via the Advanced Fellowship programme. We acknowledge support from STFC for the UK APAP network. B. C. Fawcett is thanked for his contribution in rescuing some of his original plates, and for the continuous encouragement over the years.

\section{References}

Badnell, N. R. 1997, J. Phys. B At. Mol. Phys., 30, 1

Badnell, N. R. 2011, Comput. Phys. Commun., 182, 1528

Badnell, N. R., \& Griffin, D. C. 2001, J. Phys. B At. Mol. Phys., 34, 681

Behring, W. E., Cohen, L., Doschek, G. A., \& Feldman, U. 1972, ApJ, 175, 493

Berrington, K. A., Eissner, W. B., \& Norrington, P. H. 1995, Comput. Phys.

Commun., 92, 290

Brinkman, A. C., Gunsing, C. J. T., Kaastra, J. S., et al. 2000, ApJ, 530, L111

Burgess, A. 1974, J. Phys. B At. Mol. Phys., 7, L364

Burgess, A., \& Tully, J. A. 1992, A\&A, 254, 436

Burgess, A., Chidichimo, M. C., \& Tully, J. A. 1997, J. Phys. B At. Mol. Phys., 30,33

Chidichimo, M. C., Badnell, N. R., \& Tully, J. A. 2003, A\&A, 401, 1177

Culhane, J. L., Harra, L. K., James, A. M., et al. 2007, Sol. Phys., 243, 19

Del Zanna, G. 2012, A\&A, 537, A38
Del Zanna, G., \& Mason, H. E. 2005, A\&A, 433, 731

Del Zanna, G., Berrington, K. A., \& Mason, H. E. 2004, A\&A, 422, 731

Del Zanna, G., Storey, P. J., \& Mason, H. E. 2010, A\&A, 514, A40

Del Zanna, G., Storey, P. J., Badnell, N. R., \& Mason, H. E. 2012, A\&A, 541, A90

Dere, K. P., Landi, E., Mason, H. E., Monsignori Fossi, B. C., \& Young, P. R 1997, A\&AS, 125, 149

Fawcett, B. C., Peacock, N. J., \& Cowan, R. D. 1968, J. Phys. B At. Mol. Phys., 1,295

Fawcett, B. C., Kononov, E. Y., Hayes, R. W., \& Cowan, R. D. 1972, J. Phys. B At. Mol. Phys., 5, 1255

Griffin, D. C., Badnell, N. R., \& Pindzola, M. S. 1998, J. Phys. B At. Mol. Phys., 31,3713

Hummer, D. G., Berrington, K. A., Eissner, W., et al. 1993, A\&A, 279, 298

Landi, E., Del Zanna, G., Young, P. R., et al. 2006, ApJS, 162, 261

Lemen, J. R., Title, A. M., Akin, D. J., et al. 2012, Sol. Phys., 275, 17

Malinovsky, M., Dubau, J., \& Sahal-Brechot, S. 1980, ApJ, 235, 665

Manson, J. E. 1972, Sol. Phys., 27, 107

O’Dwyer, B., Del Zanna, G., Badnell, N. R., Mason, H. E., \& Storey, P. J. 2012, A\&A, 537, A22

Storey, P. J., Del Zanna, G., Mason, H. E., \& Zeippen, C. 2005, A\&A, 433, 717

Woods, T. N., Eparvier, F. G., Hock, R., et al. 2012, Sol. Phys., 275, 115

Young, P. R., Watanabe, T., Hara, H., \& Mariska, J. T. 2009, A\&A, 495, 587 